\title{
TOUGHNESS AND FATIGUE CRACK GROWTH RATE OF TEXTURED METALS
}

\author{
J. GIL SEVILLANO \\ CEIT, Centro de Estudios e Investigaciones Técnicas de Guipúzcoa (Apdo. 1555, \\ 20080 San Sebastián), and Faculty of Engineering (University of Navarra) \\ Urdaneta 7, 20006 San Sebastián, Spain
}

(Received January 15, 1989)

The influence of anisotropy of crystallographic origin on both fracture toughness and the rate of stage-II ductile fatigue crack growth in textured metals is discussed in terms of a plane-strain small geometry change solution for plastic non-hardening materials (a Prandtl-type slip-line field solution accounting for anisotropy). Results corresponding to FCC or BCC metals sliding, respectively, on $\{111\}\langle 110\rangle$ or $\{110\}\langle 111\rangle$ systems are presented. Remarkable effects of both texture toughening and fatigue crack growth rate anisotropy are predicted. Stronger effects are anticipated in more anisotropic metals (HCP).

KEY WORDS Toughness, fatigue, crack growth, slip-line fields, FCC and BCC single crystals, anisotropic crack growth rate.

\section{INTRODUCTION}

Besides numerical attempts, modelling the crack behaviour of plastically anisotropic solids has been approached from continuum mechanics as an extension of the HRR asymptotic solutions for small scale yielding in power-law hardening materials (Pan and Shih, 1986) or by means of slip-line field solutions for contained yielding in perfectly plastic solids (Pan, 1986, Gil Sevillano and Martin Meizoso, 1988). In both cases, the material was assumed to yield according to the quadratic criterion of Hill (1950) for orthotropic materials. Recently, Gil Sevillano and Martin Meizoso (1988) studied some local aspects of static fracture on the basis of the anisotropic behaviour derived from crystal plasticity. Here, an approach combining slip-line fields and the plastic properties of the crystals as derived from the Taylor/Bishop-Hill model (multiple slip) is taken. The approach is thus strictly valid for non-hardening materials and plastic zone sizes large relative to substructure or-in the case of polycrystals-to grain size. It is developed only for small geometry change.

The use of slip line fields in the context of crystal plasticity is perfectly sound. Rice (1973) has shown that plane-strain slip-line theory is valid for any rigid-plastic material of arbitrary anisotropy provided it complies with the principle of maximum plastic work. It is thus rigorously applicable to crystals or polycrystals deforming by slip in accordance with a critical resolved shear stress (CRSS) criterion. 


\section{MODE I CRACKS IN PLASTICALLY ANISOTROPIC SOLIDS}

\section{Generalized Plane-Strain Slip-Line Fields for Plastic Flow around Sharp Cracks}

The Prandtl-type slip-line field gives a correct solution for the plane-strain plastic flow and the stress field at the vicinity of a mode I sharp crack tip in a rigid-perfectly plastic isotropic material (Rice, 1968). For orthotropic materials yielding according to the quadratic Hill's criterion, a close agreement is also found between the HRR stress fields for low-hardening material and those derived from the Prandtl slip-line field, just as it happens for the isotropic case.

For a material with arbitrary plastic anisotropy or even for an orthotropic material when the symmetry axes of the imposed stress or displacement field do not coincide with the axis or orthotropy, the orientations of the principal axes of local stresses and strains do not coincide. Consequently, the slip-line field for the contained plastic flow around a mode I crack tip can be asymmetric relative to the reference orthogonal system defined by the crack (crack growth direction, $x_{1}$, normal to crack plane, $x_{2}$ ). As shown by Rice (1973), constant state regions and centered fans are however admissible fields for every material. Consequently, the asymmetric Prandtl-type field of Figure 1a is a kinematically admissible field for the flow near a crack tip (a strain singularity results varying inversely with distance $r$ from the tip $T$ in the centered fan regions above and below the tip, as required (Rice, 1968)). Then, according to the maximum work principle (Bishop and Hill, 1951), $\theta$ is the angle leading to the minimum plastic dissipation.

The plastic dissipation for a given $\theta$ can be calculated-as described in the next section-assuming any admissible velocity field for the generalized Prandtl slip line field. This allows also for a ready derivation of the tensile stress acting normal to the crack plane ahead of the crack tip. Alternatively, as shown by Rice (1973) $\theta$ and the stresses in the slip line field can be derived from the plane-strain anisotropic yield locus of the material and the normality rule. The validity of the solution adopted here is guaranteed by the uniqueness of the stress distribution associated with the Prandtl field, despite the non-uniqueness of associated velocity fields.

\section{Solution for the Plastic Work and the Tensile Stress Level ahead of the Crack}

Figure $1 \mathrm{~b}$ shows a possible hodograph corresponding to Figure 1a. Considering the plastic dissipation per unit thickness, $B$, for the part of the field above the crack:

$$
\begin{aligned}
W / B= & \sigma \cdot \Delta \cdot \overline{T D}=u \tau(\theta)[\overline{A B}+\overline{C D}]+\int_{\theta}^{\theta+\pi / 2} \tau(\alpha) \cdot r \cdot u \cdot d \alpha \\
& +\int_{\theta-\pi / 2}^{\theta} u \cdot \tau(\alpha) \cdot r \cdot d \alpha
\end{aligned}
$$

where $\tau(\theta)$ represents the simple in-plane shear flow stress (constrained deformation) along a direction defined by $\theta$ and where use has been made of the symmetry associated to shear stresses or strains:

$$
\tau_{i j}=\tau_{j i}, \quad \varepsilon_{i j}=\varepsilon_{j i} \Rightarrow \tau(\alpha)=\tau(\alpha+\pi / 2)
$$




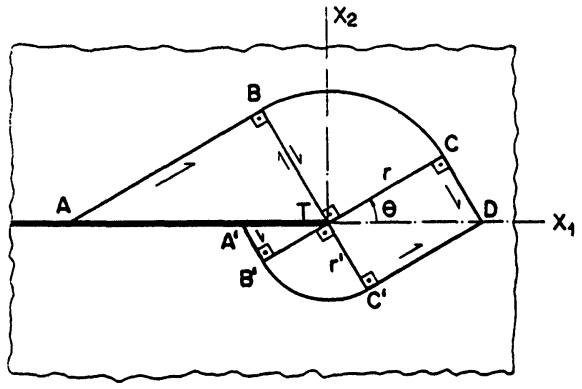

(a)

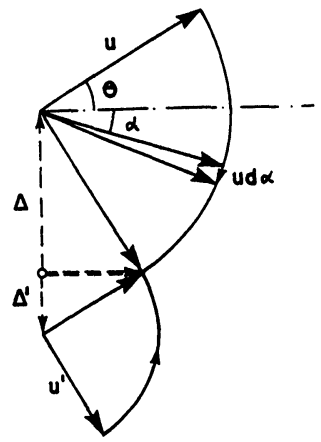

(b)

Figure 1 Asymmetric Prandtl-type slip-line field and hodograph.

$\Delta$ represents the vertical displacement of $\overline{T D}$ and $\sigma$ is the tensile stress developed normal to $\overline{T D}$.

From geometrical considerations of Figures $1 \mathrm{a}$ and $1 \mathrm{~b}$, it can be easily shown:

$$
\sigma=2 \tau(\theta) / \sin 2 \theta+\int_{\theta-\pi / 2}^{\theta+\pi / 2} \tau(\alpha) d \alpha
$$

From Eq. (2) it results that the second term of (3) is invariant on the plane considered:

$$
\sigma=2 \tau(\theta) / \sin 2 \theta+\pi \bar{\tau}
$$

where $\overline{\boldsymbol{\tau}}$ represents the average shear flow strength for simple shear along directions contained on the plane considered. $\theta$ is the angle minimizing $W / B$, i.e., minimizing $\sigma$. Obviously, the same Eq. (4) is obtained when computing the plastic work of the bottom part of the slip-line field.

Assessment of texture toughening effects. For brittle modes of static fracture, when fracture is conditioned by a critical stress level $\sigma_{f}$, the tensile stress $\sigma$ given by Eq. (4) is the most relevant parameter for assessing orientation influence. It is to be remembered, however, that $\sigma_{f}$ is also orientation dependent (Gil Sevillano and Martin Meizoso, 1988).

A crude estimation of the fracture toughness, $K_{I c}$, using a simple model of the RKR type (Ritchie, Knott and Rice, 1973) in association with this incipient slip line solution $\left(\sigma>\sigma_{f}\right.$ along a critical distance $\left.x_{c}\right)$ is sketched in Figure 2;

$$
x_{c} \simeq(\overline{T D})_{c} \propto\left(\Delta+\Delta^{\prime}\right)_{c}
$$

Lacking an analysis of the $J$ integral for the anisotropic slip line field, it appears reasonable to assume,

$$
\sigma\left(\Delta+\Delta^{\prime}\right) \approx J=K^{2} / E^{*}
$$

where the effective modulus $E^{*}$ is orientation dependent on account of elastic 


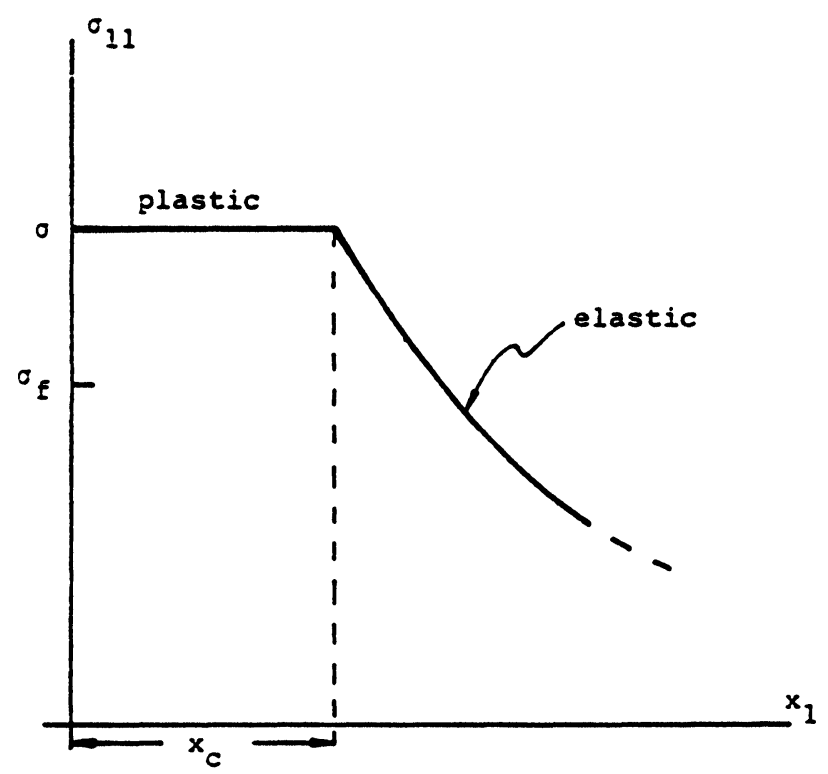

Figure 2 RKR model for brittle fracture from a sharp crack in an elastic perfectly plastic material. All the stress levels $\left(\sigma, \sigma_{f}\right.$ and the elastic solution) and, presumably, $x_{c}$, are orientation dependent. The origin is located at the crack tip and the plastic zone in $x_{1}$ direction extends along $\overline{T D}$ (Figure 1).

anisotropy (Kanninen and Popelar, 1985). Combining with Eq. (5),

$$
J_{I c}=K_{I c}^{2} / E^{*} \propto \sigma x_{c} \quad\left(\text { for } \sigma \geq \sigma_{f}\right)
$$

For ductile modes, when fracture is conditioned by a critical strain level along some distance fixed by the microstructure, the critical situation will be attained once such a distance is engulfed in the high strain region developed ahead of the blunted crack (Rice and Johnson, 1970). By similarity with the isotropic case such situation is represented by a condition formally identical with Eq. (5), that gives way to an expression for $J_{I c}$ formally identical with Eq. (7). In fact as the critical strain for local fracture is sensible to the hydrostatic stress level (also dependent on $\sigma$ ), the actual orientation dependence of $J_{I c}$ will be more complicated.

Fatigue crack growth. Two types of non phenomenological models of fatigue propagation-among the available host of them-can be distinguished: plastic blunting models and models based on damage mechanisms ahead of the crack tip (Stanzl, 1982). Actually observed fatigue crack growth rate (FCGR) probably combines contributions from both type of processes.

Both blunting and damage are susceptible of texture influence, which may, in principle, lead to either synergistic or self-cancelling effects on FCGR. From a literature survey, it appears very clearly that plastic anisotropy is a very important factor: when plastic blunting is very easy, high FCGR is observed together with ductile (striation) modes; when blunting is impeded, damage (ductile or brittle) mechanisms are promoted. But no simple criterion for assessing texture influence is at hand. The following developments apply only to the influence of crystal- 


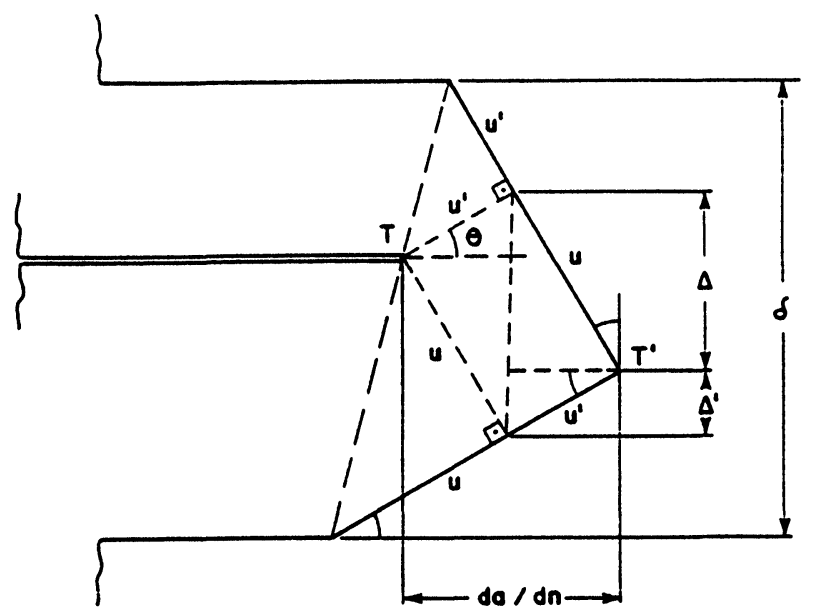

Figure 3 A possible blunting scheme corresponding to the field of Figure 1.

lographic texture on stage-II ductile fatigue crack growth by the blunting/ resharpening process.

Strong support exists for the control of ductile crack growth by the crack-tip opening displacement. Isotropic models either based on continuum mechanics, on coarse slip bands or on dislocation plasticity, lead to the proportionalities:

$$
d a / d n \propto \Delta \mathrm{CTOD} \propto \Delta J / \sigma_{y} \propto \Delta K^{2} / E \sigma_{y}
$$

where $\sigma_{y}$ is some measure of the plastic strength of the material and the other symbols have their usual meanings. The present analysis parallels that of the isotropic models. According to the plastic flow field of Figure 1, the crack tip opening displacement, CTOD, is, on account of incompressibility,

$$
\delta=\left(\Delta+\Delta^{\prime}\right)(1+\sin 2 \theta)
$$

and assuming the simplified blunting scheme of Figure 3, the crack advance per cycle is, in the absence of neither rewelding nor any kind of static or dynamic damage mechanism contributing to crack growth,

$$
d a / d n=\left(\Delta+\Delta^{\prime}\right) \sin 2 \theta
$$

Now, according to the previous estimation for $J$, Eq. (6),

$$
\sigma \cdot\left(\Delta+\Delta^{\prime}\right) \approx \Delta J=\Delta K^{2} / E^{*}
$$

Combining with Eq. (10),

$$
d a / d n \approx 2 \Delta J \sin 2 \theta / \sigma=2 \Delta K^{2} \sin 2 \theta / \sigma E^{*}
$$

\section{APPLICATION TO CUBIC METALS}

Summarizing, the contribution of plastic anisotropy to the anisotropy of toughness and FCGR is dictated by the anisotropy of the shear flow stress for fully 
constrained in-plane shear strain. For crystalline materials obeying a CRSS law, such flow stress can be calculated making use of the Taylor or Bishop-Hill procedures (Bishop and Hill, 1951). This has been done for several individual orientations of materials deforming by slip on $\{111\}\langle 110\rangle$ or $\{110\}\langle 111\rangle$ systems, i.e., FCC or BCC metals, and some results will be now presented.

Assuming uniform values of CRSS for the equivalent systems,

$$
\tau(\theta)=M(\theta) \tau_{c}
$$

where $\tau_{c}$ is the CRSS and $M(\theta)$ the "orientation factor" for shear strain in $\theta$ direction on the plane under consideration. Equations (4) and (12) can then be normalized by the CRSS:

$$
\begin{gathered}
\sigma / \tau_{c}=2 M(\theta) / \sin 2 \theta+\pi \bar{M} \\
d a / d n \propto \sin 2 \theta /\left(\sigma / \tau_{c}\right)
\end{gathered}
$$

Figure 4 shows the orientation factor for shear strain in four different crystallographic planes. Plates parallel to (001) and (011) are orthotropic; the symmetry of the shear strain tensor (Eq. (2)) introduces a new symmetry plane at $45^{\circ}$ of the crystallographic symmetry plane. A plate parallel to (111) does not possess orthotropy, but behaves orthotropically for in-plane shear strains. Orientation (123) is a general, non-symmetric case.
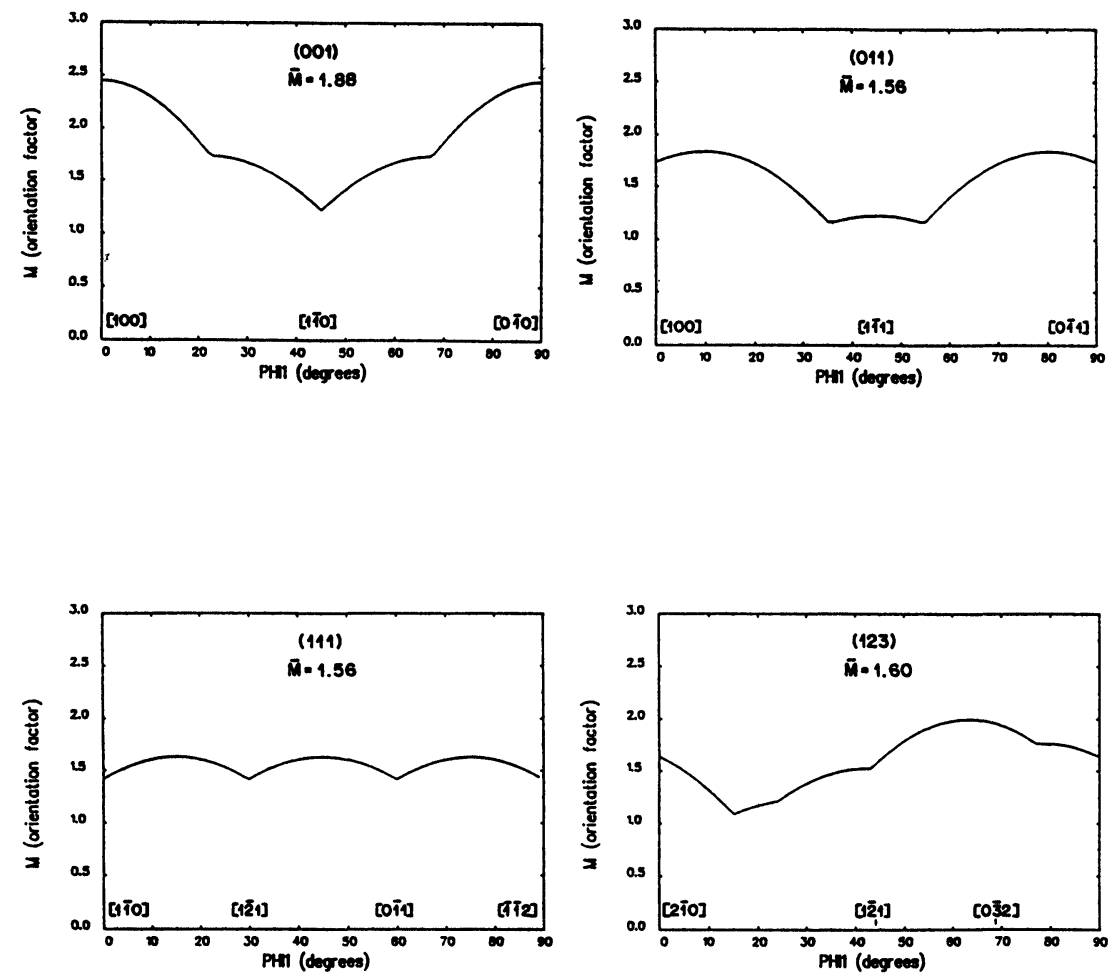

Figure 4 Orientation factors for in-plane simple shear strain for four different (hkl) planes. FCC or BCC metals, Bishop-Hill model. 


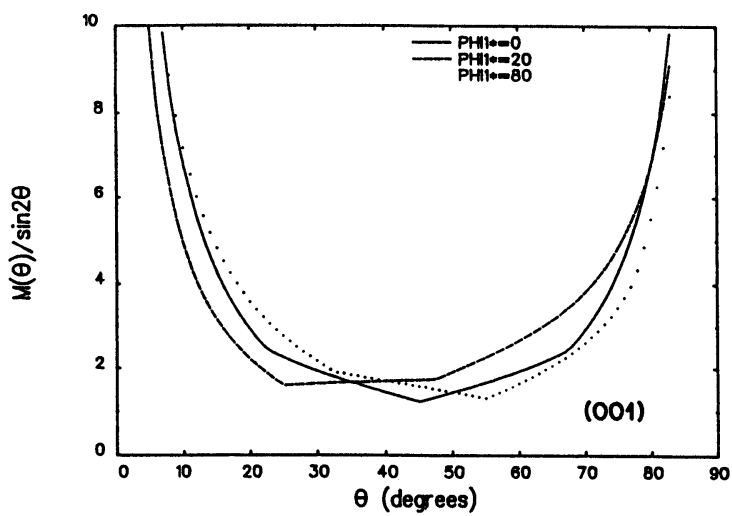

Figure 5 Search for the minimum plastic work. Three examples of the effect of misorienting the slip-line field for orientations of the crack on (001) plane.

For a random aggregate of cubic crystals, the orientation factor for shear strain is 1.66 (Hosford, 1973). The maximum shear flow stress in a crystal is $\sqrt{6} \tau_{c}=2.45 \tau_{c}$ and the minimum is obviously $\tau_{c}$ (imposed strain coincident with crystallographic slip strain). The plastic anisotropy is thus far from negligible. The maximum in-plane shear strength difference is $100 \%$ (e.g., in the (001) plane).

Figure 5 presents three examples of the search for minimum plastic work for edge cracks in a plate with "cube texture". Only the first term of Eq. (14) must be accounted for, the other term being independent of the orientation of the slip-line field relative to the crack. The examples of the figure correspond to three different orientations of the crack ( $x_{1}$ axis) defined by the angle $\varphi_{1}$ around the normal to the plane (hkl) studied, as indicated in the figures $\left(\varphi_{1}\right.$ is one of the Euler angles-Bunge convention (Bunge, 1982)-defining the crystal orientation relative to the external reference system associated to the crack, Figure 1). $M\left(\varphi_{1}\right)$ presents vertices owing to the polyhedric nature of the crystal yield locus and this causes discontinuities in the $\theta\left(\varphi_{1}\right)$ curve. Figure 6 presents such a curve for a

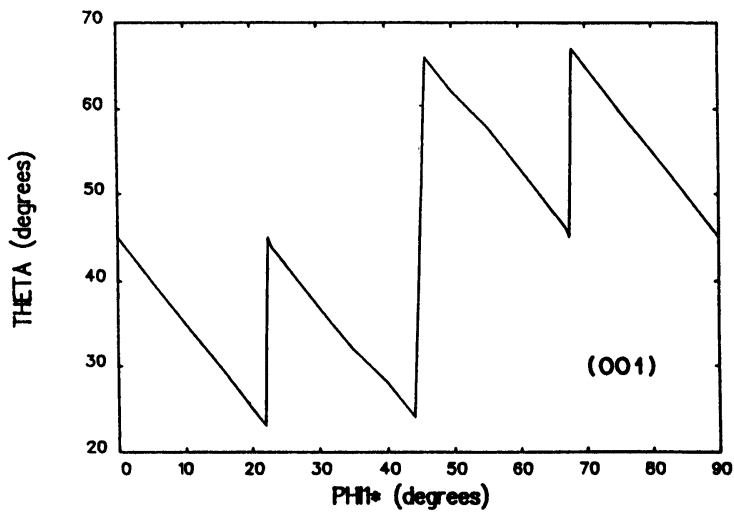

Figure 6 Orientation of the slip-line field for different orientations of cracks transverse to a (001) plane (FCC or BCC metals). 


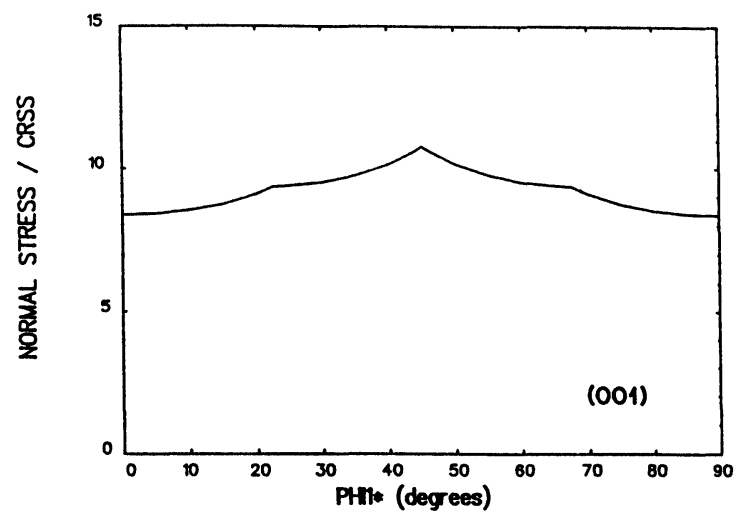

Figure 7 Tensile stress level ahead of cracks transverse to a (001) plane in FCC or BCC metals as a function of the in-plane orientation of crack advance.

plate with "cube" texture. It has been calculated with the data contained in Figure $4 \mathrm{a}$. It may be seen that the asymmetry predicted for the slip-line field can be very strong, $\theta$ varying from $22.5^{\circ}$ to $67.5^{\circ}$. However, the influence of orientation on the tensile stress level ahead of the crack- $\sigma / \tau_{c}$, Eq. (14)-is only moderate, Figure 7, with just a 30\% difference between the maximum and the minimum for a plane with such a strong shear strength anisotropy as the (001) plane. The weaker anisotropy of toughness relative to the plastic anisotropy could be anticipated from inspection of Eq. (14), where the second termcorresponding to the $\pi / 2$ centered fans of the slip line field-averages the in-plane shear flow stress of the plate.

\section{TWO ILLUSTRATIVE CASE STUDIES}

\section{Propagation and Arrest of Cleavage Cracks on \{001\} Planes of BCC Metals}

The tensile stress ahead of $\{001\}$ cracks growing along different directions lying on this BCC cleavage plane (calculated according to the model presented in this paper) shows a maximum for $\langle 100\rangle$ orientations and a minimum for $\langle 110\rangle$ orientations (Figure 8). For very brittle situations (i.e., very low temperature or high strain rates, $\sigma \gg \sigma_{f}$ ), in the absence of elastic anisotropy effects, the critical condition for crack propagation on single crystals or cube textured polycrystals loaded along $\langle 001\rangle$ direction will first be reached for crack growth in $\langle 110\rangle$ orientation (minimum toughness) when an RKR type criterion is to be met. However, for cracks with sharp tips (e.g., cracks running dynamically, propagating by cyclic cleavage or SCC, where some plasticity is however observed, Argon, 1987, Argon et al., 1988), the tensile stress at the crack tip will be the highest for $\langle 100\rangle$ direction of growth. Finally, plastic blunting of $\{001\}$ cracks requires the lowest energy for $\langle 110\rangle$ growth direction. Consequently, the arrest fronts of brittle (cleavage) cracks of $\mathrm{BCC}$ metals are expected to be aligned parallel to $\langle 110\rangle$ directions.

The last assertion is confirmed by the experimental observation of arrested $\{001\}$ internal square cracks with sides parallel to the $\langle 110\rangle$ directions in $\mathrm{Fe}-3 \% \mathrm{Si}$ 


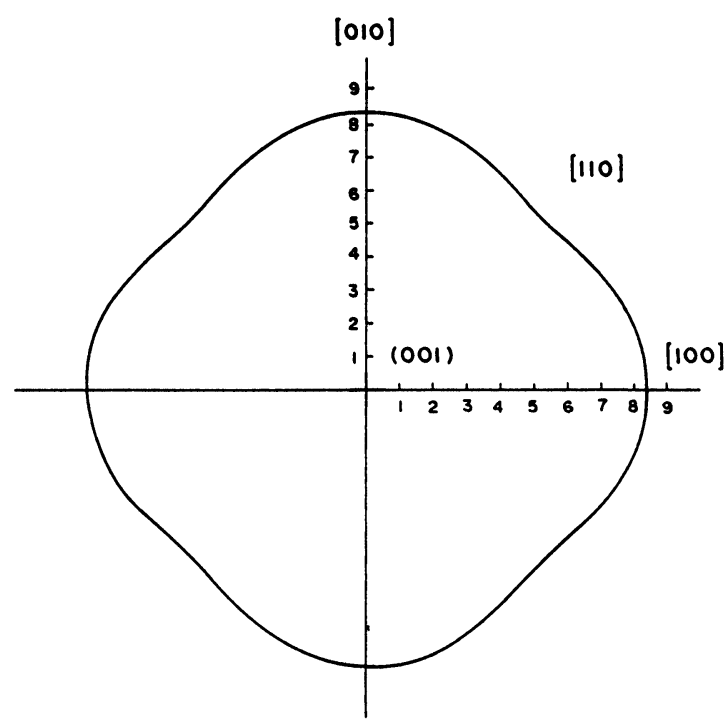

Figure 8 Polar plot of $\sigma / \tau_{c}$, the tensile stress (relative to the CRSS) normal to the crack plane ahead of the crack tip, for cracks growing along different directions on $\{001\}$ planes of cubic metals. Slip line fields are symmetrically oriented $\left(\theta=45^{\circ}\right)$.

crystals. The cracks grew in bursts produced by hydrogen cracking (Gell and Robertson, 1964). Such crack shape was attributed by Atkinson and Head (1966) to the effect of elastic anisotropy on the critical internal pressure for crack propagation: $\langle 110\rangle$ is the direction which needs the greatest pressure to start propagation and $\langle 100\rangle$ that which needs to lowest pressure, assuming perfect elastic behavior. However, the observed square cracks are arrested cracks, with evidence of associated intense plasticity at their tips. It appears more natural to explain their shape on the basis of the anisotropy of the critical condition for arrest, determined by plastic blunting. Additional support for the present explanation is given by the observation of $\langle 110\rangle$ crack fronts during the slow (presumably discontinuous) propagation of $\{100\}$ cleavage cracks in tungsten at temperatures close to the ductile to brittle transition (Hull et al., 1965). Tungsten is elastically isotropic.

Fatigue Crack Growth for $\{001\}$ and $\{110\}$ Cracks

$\{001\}$ cracks. Figure 8 represents the polar plot of the inverse of the FCGR for cracks growing along any direction on the $\{001\}$ plane, according to Eq. (15), i.e. in the absence of any damage contribution to crack growth, assuming plastic blunting and resharpening as the only contributing mechanism. Direction $\langle 110\rangle$ is the energetically most favourable orientation of the crack front for ductile crack advance and, indeed, a strong tendency to make the crack front of a fatigue crack parallel to $\langle 110\rangle$ is found when testing $\{001\} \mathrm{Cu}$ single crystals (Newmann, 1974) or aluminium polycrystals (Pelloux, 1969). Driver and coworkers (Driver, 1979) have also found that $\{100\}\langle 110\rangle$ is a preferential path for ductile fatigue crack propagation in $\mathrm{BCC}$ alloys. 


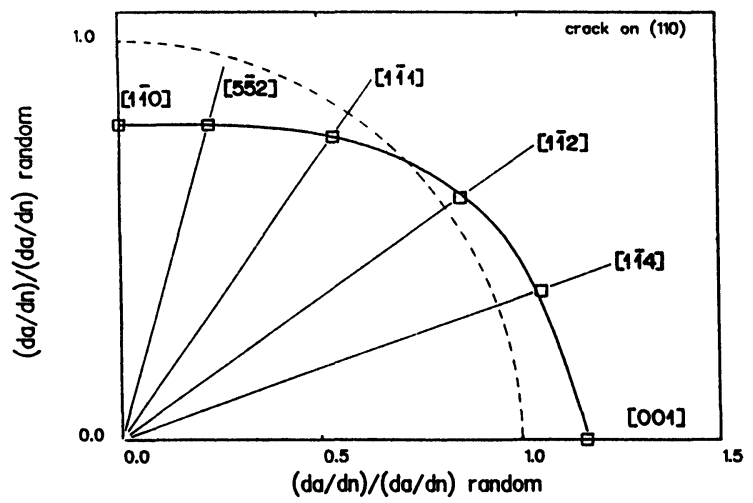

Figure 9 Predicted growth rate of cracks on the (110) plane of an FCC or BCC material under mode I loading conditions. Polar diagram.

$\{110\}$ cracks. Finally, the relative mode I FCGR predicted with the same premises for cracks on $\{110\}$ planes is shown in Figure 9 in the form of a polar diagram. Owing to the particular symmetry of this orientation, the results correspond to symmetrically oriented slip-line fields, as in the previous case $\left(\theta=45^{\circ}\right)$. The relative rate of crack propagation is maximum in $\langle 100\rangle$ directions and minimum in $\langle 110\rangle$ directions. Some experimental results in agreement with this theoretical prediction have been published (Rieux et al., 1978, Driver, 1979, Lismont, 1986; Roux et al., 1986).

\section{CONCLUSIONS}

- An attempt to incorporate crystal plasticity into plane strain fracture modelling has been presented. It allows quantitative exploration of the influence that texture has on fracture anisotropy.

- The predictions of the model for some illustrative examples of application are in agreement with experimental results.

- Only the contribution of crystalline plasticity to fracture anisotropy has been modelled. In real cases, the contributions of elastic anisotropy and-for FCG - of damage mechanisms can obscure the influence of plastic anisotropy.

\section{ACKNOWLEDGEMENT}

The author acknowledges financial support from the Departamento de Economía, Diputación Foral de Guipúzcoa, Basque Country, Spain.

\section{References}

Argon, A. S. (1987), Acta Metall, 35, 1985.

Argon, A. S., Deng, D., Thompson, R. and Lin, I. H. (1988), Acta Metall., to be published.

Atkinson, C. and Head, A. K. (1966), Int. J. Fracture, 2, 489. 
Bishop, J. F. W. and Hill, R., (1951), Phil. Mag., 42, 414.

Bunge, H. J. (1982), Texture Analysis in Materials Science, Butterworths, London.

Driver, J. (1979). Discussion to the paper by Newmann et al., "Fatigue Mechanisms", p. 371, ASTM STP 675, American Society for Testing of Materials.

Gell, M. and Robertson, W. D. (1964), Acta Metall, 12, 108.

Gil Sevillano, J. and Martin Meizoso, A. (1988), Proc. ICOTOM 8, p. 897. J. S. Kallend and G. Gottstein, eds., The Metallurgical Society, AIME.

Hill, R. (1950), The Mathematical Theory of Plasticity. Clarendon Press, Oxford.

Hosford, W. F. (1973), Metall. Trans., 4, 1416.

Hull, D., Beardmore, P. and Valintine, A. P. (1965), Philos. Mag., 12, 1021.

Kanninen, M. F. and Popelar, C. H. (1985), Advanced Fracture Mechanics. Oxford University Press, Oxford.

Lismont, H. (1986), Eng. thesis (Eindwerk), Dept. of Metallurgy and Materials Engineering, University of Leuven (Belgium).

Newmann, P. (1974), Acta Metall., 22, 1155.

Pan, J. (1986), J. Mech. Phys. Solids, 34, 617.

Pan, J. and Shih, C. F. (1986), Mechanics of Materials, 5, 299.

Pelloux, R. M. N. (1969), Trans. ASM, 62, 281.

Rice, J. R. (1968), J. Appl. Mechanics, Trans. ASME, 35, 379.

Rice, J. R. (1973), J. Mech. Phys. Solids, 21, 63.

Rice, J. R. and Johnson, M. A. (1970), Inelastic Behaviour of Solids, p. 641, M. F. Kanninen et al., eds., McGraw-Hill, New York.

Rieux, P., Driver, J. and Rieu, J. (1978). Mem. Sci. Rev. Metall., 75, 423.

Ritchie, R. O., Knott, J. F. and Rice, J. R. (1973), J. Mech. Phys. Solids, 21, 395.

Roux, R., Charrier, J. and Gasc, C. (1986), Strength of Metals and Alloys, vol. 2, p. 1323. H. J. McQueen et al., eds., Pergamon Press, Oxford.

Stanzl, S. A. (1982), Res. Mechanica, 5, 241. 